\title{
Superconducting gap and vortex lattice of the heavy-fermion compound $\mathrm{CeCu}_{2} \mathrm{Si}_{2}$
}

\author{
Mostafa Enayat, ${ }^{1}$ Zhixiang Sun, ${ }^{1}$ Ana Maldonado, ${ }^{1,2}$ Hermann Suderow, ${ }^{3,4}$ Silvia Seiro, ${ }^{5}$ Christoph Geibel, ${ }^{5}$ \\ Steffen Wirth, ${ }^{5}$ Frank Steglich, ${ }^{5}$ and Peter Wahl ${ }^{1,2, *}$ \\ ${ }^{1}$ Max-Planck-Institut für Festkörperforschung, Heisenbergstrasse 1, D-70569 Stuttgart, Germany \\ ${ }^{2}$ SUPA, School of Physics and Astronomy, University of St. Andrews, North Haugh, St. Andrews, Fife, KY16 9SS, United Kingdom \\ ${ }^{3}$ Laboratorio de Bajas Temperaturas, Departamento de Física de la Materia Condensada, Instituto de Ciencia de Materiales Nicolás \\ Cabrera, Condensed Matter Physics Center (IFIMAC), Facultad de Ciencias Universidad Autónoma de Madrid, 28049 Madrid, Spain \\ ${ }^{4}$ Unidad Asociada de Bajas Temperaturas y Altos Campos Magnéticos, UAM, CSIC, Cantoblanco, E-28049 Madrid, Spain \\ ${ }^{5}$ Max-Planck-Institut für die Chemische Physik fester Stoffe, Nöthnitzer Strasse 40, 01187 Dresden, Germany \\ (Received 1 May 2015; revised manuscript received 17 December 2015; published 20 January 2016)
}

\begin{abstract}
The order parameter and pairing mechanism for superconductivity in heavy-fermion compounds are still poorly understood. Scanning tunneling microscopy and spectroscopy at ultralow temperatures can yield important information about the superconducting order parameter and the gap structure. Here, we study the first heavyfermion superconductor, $\mathrm{CeCu}_{2} \mathrm{Si}_{2}$. Our data show the superconducting gap which is not fully formed and exhibits features that point to a multigap order parameter. Spatial mapping of the zero-bias conductance in magnetic field reveals the vortex lattice, which allows us to unequivocally link the observed conductance gap to superconductivity in $\mathrm{CeCu}_{2} \mathrm{Si}_{2}$. The vortex lattice is found to be predominantly triangular with distortions at fields close to $\sim 0.7 H_{c 2}$.
\end{abstract}

DOI: 10.1103/PhysRevB.93.045123

\section{INTRODUCTION}

Superconductivity in heavy-fermion materials was first observed in $\mathrm{CeCu}_{2} \mathrm{Si}_{2}$ [1] and was unexpected. The formation of heavy-fermion bands is usually ascribed to the interaction between delocalized conduction electrons and localized magnetic moments, whereas localized magnetic moments in conventional superconductors rapidly destroy superconductivity. Since this discovery, superconductivity has been found in a range of other heavy-fermion materials, often in close proximity to a quantum phase transition between a magnetically ordered phase and a phase dominated by Kondo screening. This proximity of superconductivity to a magnetic quantum critical point found in many Ce-based compounds [2] indicates that magnetic fluctuations and the influence of the quantum critical point might promote superconductivity in these materials. The physics near the quantum critical point sensitively depends on the balance between screening of the local magnetic moments and interactions between them [3]. In $\mathrm{CeCu}_{2} \mathrm{Si}_{2}$, slight changes in the exact composition (specifically the $\mathrm{Cu}$-to-Si ratio) result in superconducting crystals ( $S$ type), magnetically ordered samples ( $A$ type), or samples which exhibit competing phases ( $A / S$ type) $[4,5]$. Superconductivity occurs close to a spindensity-wave-type quantum critical point and is expected to be unconventional in nature. Neutron scattering data indicate that superconductivity is mediated by spin fluctuations rather than by phonons [6]. Despite intense research, the precise form of the superconducting order parameter remains elusive. Measurements of the relaxation rate of the nuclear quadrupolar resonance [7,8] and specific heat under pressure [9] have yielded evidence for an unconventional order parameter. Angle-resolved resistivity measurements of the upper critical field $H_{\mathrm{c} 2}$ exhibit a fourfold symmetry of $H_{\mathrm{c} 2}$ consistent with a $d_{x y}$ symmetry of the order parameter [10]. In contrast, recent thermodynamic measurements offer evidence for nodeless

*wahl@st-andrews.ac.uk multiband superconductivity in $\mathrm{CeCu}_{2} \mathrm{Si}_{2}$, challenging the view that the pairing symmetry is of nodal $d$-wave type [11]. Also from theory, different symmetries of the order parameters and different coupling mechanisms have been proposed, e.g., a $d$-wave symmetry for superconductivity mediated by magnetic fluctuations [12] and, very recently, $s_{ \pm}$-wave symmetry emerging from magnetic fluctuations of higher order [13].

Scanning tunneling microscopy (STM) and spectroscopy (STS) has been successfully employed to study heavy-fermion materials $[14,15]$ as well as Kondo lattice compounds [16,17]. Yet, due to the low temperatures required for these experiments, STM and STS have only been applied to few heavyfermion superconductors successfully [18-23], despite these having been thoroughly studied using macroscopic techniques (see, e.g., Ref. [24] for a review). In particular, quasiparticle interference imaging provides evidence for a $d_{x^{2}-y^{2}}$ pairing symmetry in $\mathrm{CeCoIn}_{5}[22,23]$. Imaging the vortex lattice and transitions in the vortex lattice arrangement can yield additional insight into the structure of the superconducting gap [22]. Here we use a dilution-refrigerator-based STM to study the superconducting properties of $\mathrm{CeCu}_{2} \mathrm{Si}_{2}$ at temperatures down to $20 \mathrm{mK}$ [25]. Experiments have been performed on an $S$-type single crystal with a superconducting transition temperature $T_{\mathrm{c}}=0.59 \mathrm{~K}$ and an upper critical field along $c$ of $\mu_{0} H_{\mathrm{c} 2} \sim 2.3 \mathrm{~T}$.

\section{EXPERIMENTAL METHODS AND SAMPLE PREPARATION}

$\mathrm{CeCu}_{2} \mathrm{Si}_{2}$ crystallizes in the $\mathrm{ThCr}_{2} \mathrm{Si}_{2}$ I4/ $\mathrm{mmm}$ bodycentered tetragonal structure with lattice parameters $a=$ 4.1031(2) $\AA$ and $c=9.9266(5) \AA$ [5] [see Fig. 1(a)]. Many materials with the same crystal structure cleave easily perpendicular to the crystallographic $c$ axis (e.g., the 122 iron pnictides, $\mathrm{YbRh}_{2} \mathrm{Si}_{2}, \mathrm{CeRh}_{2} \mathrm{Si}_{2}, \mathrm{YbCo}_{2} \mathrm{Si}_{2}$, and $\mathrm{URu}_{2} \mathrm{Si}_{2}$ ), due to differences in the bond strengths favoring breakage of the crystal within specific planes. $\mathrm{CeCu}_{2} \mathrm{Si}_{2}$ crystals do not cleave in a way which yields extended, atomically flat 
(a)
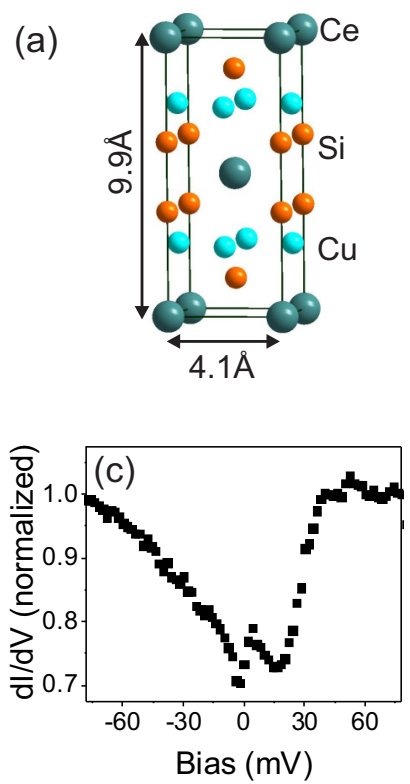

(b)
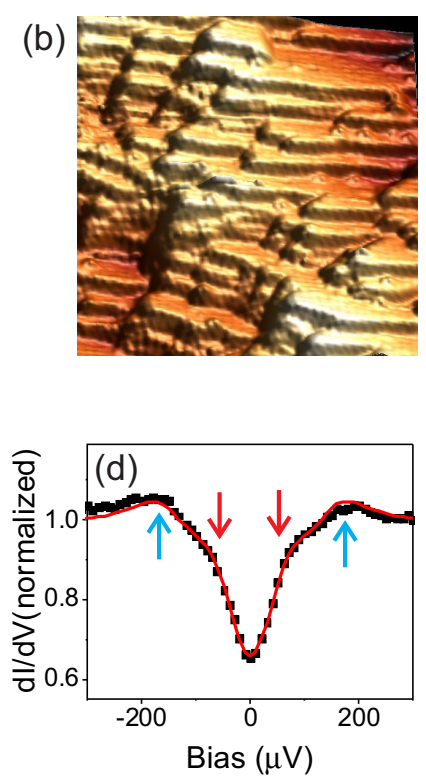

FIG. 1. (a) Body centered tetragonal crystal structure of $\mathrm{CeCu}_{2} \mathrm{Si}_{2}$. (b) Topographic image of the surface after cleavage showing terraces which exhibit atomic periodicities and step heights of $\sim 2 \AA\left(30 \times 30 \mathrm{~nm}^{2}, T=4.5 \mathrm{~K}\right)$. (c) High energy normalized tunneling spectrum taken at $T=200 \mathrm{mK}$ showing a W-shaped feature close to the Fermi energy $\left(V_{\mathrm{rms}}=3 \mathrm{mV}\right)$. (d) Normalized tunneling spectrum recorded at $20 \mathrm{mK}\left(V_{\mathrm{rms}}=40 \mu \mathrm{V}\right)$, exhibiting a gaplike feature with two shoulders symmetric with respect to zero bias. Blue arrows indicate the main gap, and red arrows the low energy shoulder. The red line shows the best fit obtained for two gaps, one of $d$-wave symmetry at low energies and one of $s$-wave symmetry at high energies (for details see text and Ref. [28]).

terraces. The bonds within the unit cell appear to be of rather similar strength, which prevents the development of a preferred cleaving plane [26]. Out of more than 30 single crystals which we cleaved, only two resulted in images which exhibit atomic periodicity and atomically flat terraces as reported below.

To promote cleaving in the $a b$ plane, a groove was cut around the perimeter of the sample in a plane perpendicular to the $c$ axis. In order to avoid surface contamination the sample was cleaved in situ in cryogenic vacuum at a cleaving stage fixed to the $4 \mathrm{~K}$ plate of the cryostat. After cleaving, the sample was immediately transferred into the STM head. Differential conductance spectra were measured with a lock-in amplifier, with a bias modulation between 10 and $40 \mu \mathrm{V}_{\text {rms }}$ (unless stated otherwise). The base temperature of the instrument is below $20 \mathrm{mK}$; the electronic temperature of the instrument has been determined previously to be $137 \mathrm{mK}$ [25].

\section{RESULTS}

\section{A. Imaging}

After successful cleavage of the sample, flat regions in an area of several hundred nanometers were found. In the absence of a natural cleavage plane, different cleaves result in different surface terminations, and also across the sample, the termination is not the same everywhere. Figure 1(b) shows an example of atomically flat terraces with steps of a height of

$2 \pm 0.2 \AA$ in between. For the specific area shown in Fig. 1(b), we can analyze the atomic periodicity and tilt angle and can attribute it to a (105) surface. Spectra taken in a range of $\pm 80 \mathrm{mV}$ show a W-shaped feature close to the Fermi level [see Fig. 1(c)], reproducible across different surface terminations. It is suggestive to interpret the peaklike feature near zero bias as a signature of the heavy $4 f$ bands; its position is consistent with the energy of the Kondo resonance determined from angular resolved photoemission [27].

\section{B. Spectroscopy of the Superconducting gap}

At the lowest temperature achieved, $20 \mathrm{mK}$, the differential tunneling conductance measured on this surface reveals a gaplike feature on an energy scale of $100 \mu \mathrm{eV}$ which we attribute to the superconducting gap [see Fig. 1(d)]. The spectra do not show a strong dependence on the location on the surface. The tunneling spectrum of the superconducting gap exhibits a rather complex structure with weak coherence peaks at $\pm 170 \mu \mathrm{V}$, additional shoulders at $\pm 75 \mu \mathrm{V}$, and a substantial zero-bias conductance of about $65 \%$ of the normal state differential conductance. We have attempted to describe the data by different models for the superconducting gap (for details of the fits see [28]). We find excellent agreement for two gaps, one which describes the low energy features by a superconducting gap with a $d$-wave symmetry and a second one describing the high energy features by a gap with $s$-wave symmetry. The extracted fitting parameters give $\Delta_{1}=(56 \pm 3) \mu \mathrm{eV}$ for the $d$-wave gap and $\Delta_{2}=(147 \pm 4) \mu \mathrm{eV}$ for the $s$-wave gap. These findings point towards a rather complex multigap superconducting order parameter. The ratio of the gap magnitudes of the large and the small gap obtained from the best fit to the tunneling data $\Delta_{2} / \Delta_{1} \approx 2.6$ compares well with the ratio of 2.5 deduced from specific heat [11]. The shape of the tunneling spectra is similar to those observed in $\mathrm{CeCoIn}_{5}[22,23]$.

Figure 2(a) shows for comparison tunneling spectra taken at $T=200 \mathrm{mK}\left(<T_{\mathrm{c}}\right)$ at zero magnetic field and at a field of $\mu_{0} H=2 \mathrm{~T}$ parallel to $c$, close to the upper critical field $H_{\mathrm{c} 2}$ [5]. As can be seen from the spectrum measured at $2 \mathrm{~T}$, the gap has almost closed completely. The temperature dependence of the spectra as shown in Fig. 2(b) reveals that the gap vanishes as the temperature rises from 20 to $600 \mathrm{mK}$.

To assess the temperature dependence of the gap size in a more quantitative way, we have used the Dynes equation [29] to fit the spectra in Fig. 2(b) with a single isotropic $s$-wave gap. While a multigap order parameter would be more appropriate, the fit becomes unstable as the features of the spectra become broadened at higher temperatures. The broadening parameter $\Gamma$ has been fixed to its value at base temperature, $\Gamma=71 \mu \mathrm{eV}$ and is likely dominated by gap anisotropy and the multigap structure. In addition we have accounted for broadening due to the finite temperature and resolution. While the fits yield overall good agreement with the data, there are also clear deviations, especially for the spectra obtained at the lowest temperatures. We attribute both the fit quality and the large broadening parameter to the fact that the superconducting order parameter in $\mathrm{CeCu}_{2} \mathrm{Si}_{2}$ is more complex than just a single $s$-wave gap. We expect the characteristic gap size extracted in this way to be qualitatively representative of the behavior of the true gap. The temperature dependence of the gap size 

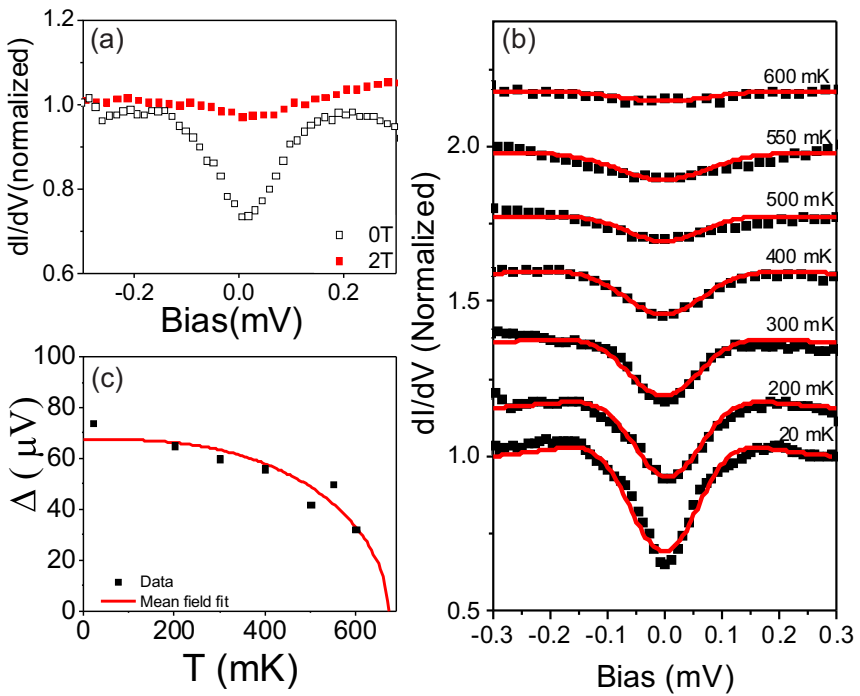

FIG. 2. (a) Differential conductance spectra (normalized at $-0.4 \mathrm{mV}$ recorded at $200 \mathrm{mK}$ in zero field (open black symbols) and in a field of $2 \mathrm{~T}$ (solid red symbols). (b) Scanning tunneling spectroscopy as a function of temperature $\left(V_{\mathrm{rms}}=24 \mu \mathrm{V}\right.$, except at $20 \mathrm{mK} V_{\mathrm{rms}}=40 \mu \mathrm{V}$ ). Red lines represent fits with the Dynes equation for a single $s$-wave gap [29]; spectra have been shifted vertically for clarity. (c) Gap size as a function of temperature extracted from fits in (b); the red line represents the mean-field behavior expected for an $s$-wave order parameter, $\Delta(T)=\Delta_{0} \tanh \left(\frac{\pi}{2} \sqrt{\frac{T_{\mathrm{c}}}{T}-1}\right)$. The fit yields $\Delta_{0}=67 \pm 3 \mu \mathrm{eV}$ and $T_{\mathrm{c}}=670 \pm 30 \mathrm{mK}$.

$\Delta(T)$ obtained in this way is shown in Fig. 2(c). It reveals similarity to a mean-field BCS behavior (red solid line) [30]. Extrapolation of the gap size to $0 \mathrm{~K}$ [see Fig. 2(c)] yields a value of $67 \pm 3 \mu \mathrm{eV}$, slightly smaller than what is found from an isotropic $s$-wave fit at base temperature $(\Delta=71 \mu \mathrm{eV}$ at $20 \mathrm{mK}$ ). The difference of the temperature dependence of the gap size for $s$-wave and $d$-wave order parameters is rather minor, so that we would not expect to be able to distinguish them from the temperature dependence of the gap size [31].

\section{Imaging of the vortex lattice}

Spatial maps of the zero-bias tunneling conductance (ZBTC) at finite magnetic fields can reveal the vortex lattice [32]. Observation of the vortex lattice can provide further information on the order parameter. For isotropic order parameter and electronic structure, the vortices usually arrange themselves in a triangular Abrikosov lattice [33]. Other symmetries of the vortex lattice can occur if there is an anisotropic interaction between the vortex cores, e.g., due to the symmetry of the superconducting order parameter or anisotropies of the electronic structure [34-36]. Maps of the differential conductance at zero bias were taken at magnetic fields of $\mu_{0} H=0.5,1,1.5,1.6,1.7 \mathrm{~T}$ [see Figs. 3(a)-3(e)]. As expected, the number of vortices is proportional to the applied magnetic field. Despite the rather rough surface morphology, our data show an ordered vortex lattice which is close to triangular symmetry.

We have analyzed the distance between the vortex cores $a_{i}$ from the autocorrelation of the ZBTC maps along the high
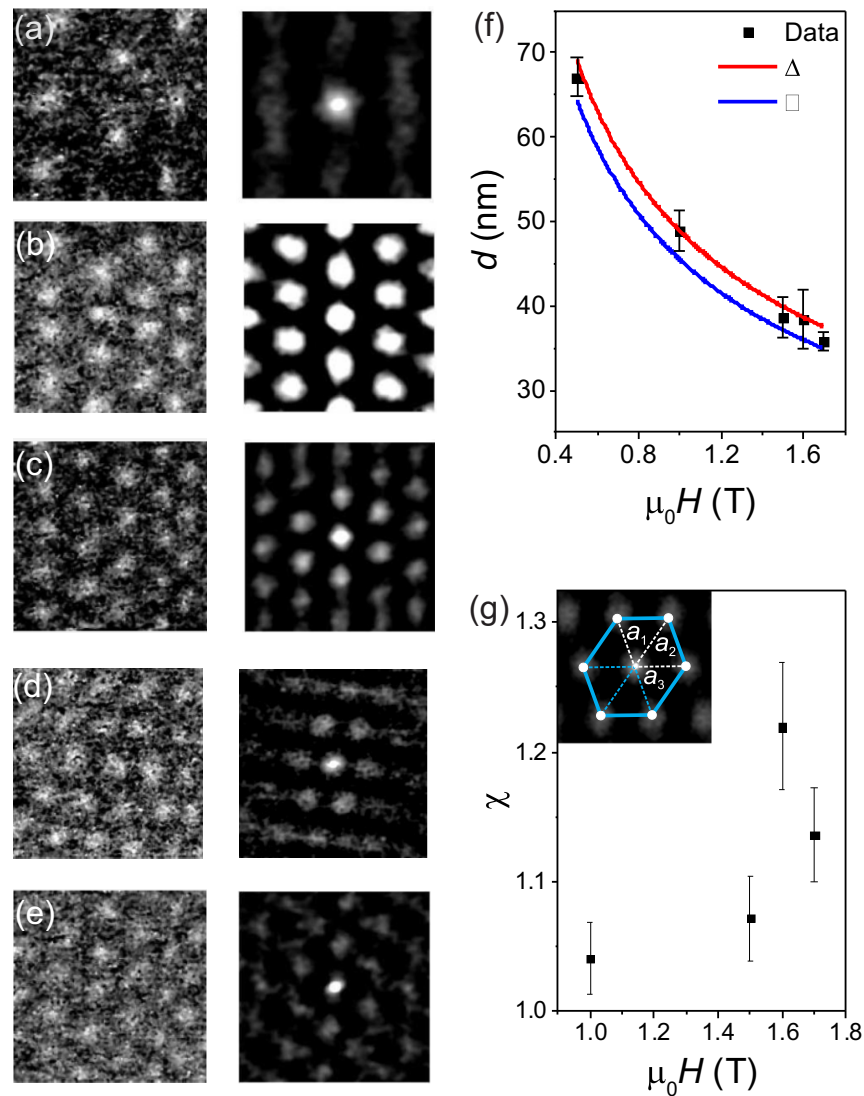

FIG. 3. (a)-(e) Maps of the zero-bias tunneling conductance at $0.2 \mathrm{~K}\left(150 \times 150 \mathrm{~nm}^{2}, V_{\mathrm{rms}}=40 \mu \mathrm{V}\right)$ taken after cooling the sample in zero field as a function of magnetic field $(0.5,1,1.5,1.6$, and $1.7 \mathrm{~T})$, together with the autocorrelation. The data have been filtered for better contrast. The triangular vortex lattice is clearly seen. (f) Distance between the vortex cores as a function of magnetic field. The points are obtained from averaging the distances in the three high symmetry directions from the autocorrelation. Solid lines show the expected dependencies for a triangular (red line) and square lattice (blue line). (g) Anisotropy of the vortex lattice extracted from the autocorrelation of differential conductance maps as a function of magnetic field. As a measure for the anisotropy, we use $\chi$ [see Eq. (1); inset shows the anisotropy exemplarily for the autocorrelation of the map taken at $1.6 \mathrm{~T}$ ]. Maps taken with a tunneling set point of $U=5 \mathrm{mV}$ [except (d) with $U=3 \mathrm{mV}], I=0.6 \mathrm{nA}$.

symmetry directions of the vortex lattice. The average distance between the neighboring vortices as a function of magnetic field reveals a behavior consistent with a triangular lattice [Fig. 3(f)]. As can be seen from Figs. 3(a)-3(e), our data indicate a distortion of the vortex lattice away from regular triangular symmetry, which becomes largest at magnetic fields of 1.6 T. At this field, the vortex lattice also appears with a different orientation than at smaller or larger fields. We define

$$
\chi=\max \left(a_{1}, a_{2}, a_{3}\right) / \min \left(a_{1}, a_{2}, a_{3}\right)
$$

as a measure for the asymmetry of the vortex lattice, with $\chi=1$ corresponding to a regular triangular vortex lattice [see inset in Fig. 3(g)]. The magnetic field dependence of $\chi$ is plotted in Fig. 3(g), confirming a significant distortion of the vortex lattice specifically at a field of $1.6 \mathrm{~T}$ [37]. 

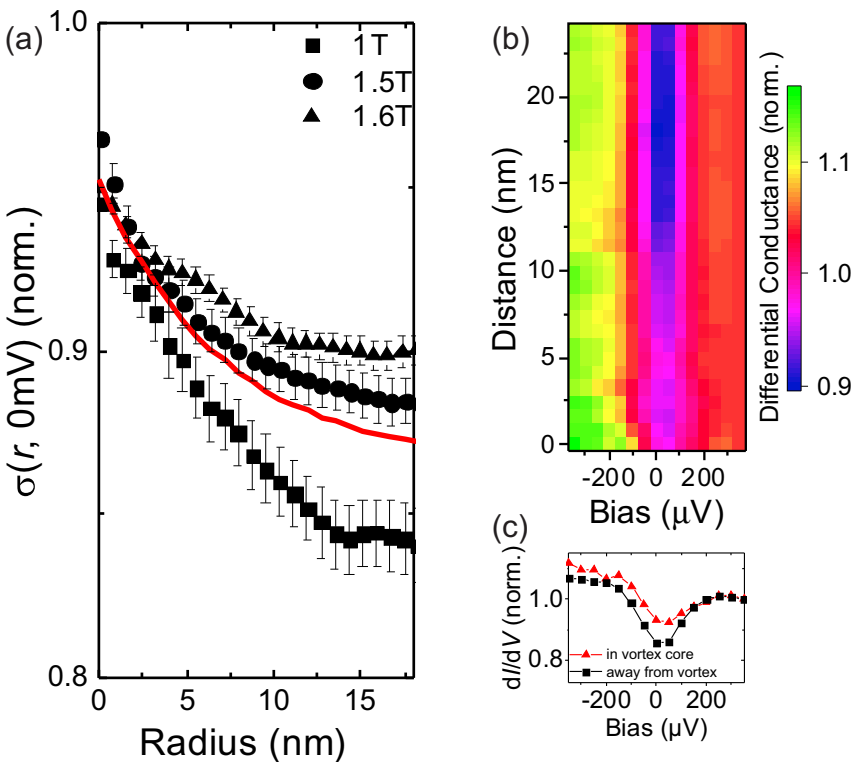

FIG. 4. (a) Evolution of the azimuthal average of the normalized $\mathrm{ZBTC} \sigma(r, 0)$ at $0.2 \mathrm{~K}$ and $1,1.5$, and $1.6 \mathrm{~T}$ as a function of the radial distance $r$ from the vortex center (error bars represent the standard deviation of the data, average over multiple vortex cores). From fits of Eq. (2) to the individual curves we obtain $\xi=7.1 \pm 0.3 \mathrm{~nm}$ on average (solid line). (b) Azimuthally averaged tunneling spectra as a function of distance from a vortex core measured at $1 \mathrm{~T}$. The zero-bias tunneling conductance is substantially larger inside the vortex core than away from the vortex core (data has been median filtered for clarity, spectra normalized at a bias voltage of $350 \mu \mathrm{V}$ ). (c) Tunneling spectra from (b) in the vortex core (red triangles) and away from a vortex core (black squares). Even in the vortex core, the zero-bias tunneling conductance stays below the normal state value (i.e., at larger bias voltages).

This distortion is robust across multiple data sets taken with different parameters, excluding drift as a possible reason. Also the surface roughness is unlikely the cause for the anisotropy, because this is similar for the images in Figs. 3(a)-3(e).

\section{Spectroscopy of individual vortex cores}

Further insight into the properties of superconductivity can be obtained by analyzing spectra taken on individual vortex cores. In Fig. 4(a), we show the decrease of the zero-bias conductance, $\sigma(r, 0)$, as a function of distance $r$ from the center of the vortex core, as superconductivity recovers. The characteristic length scale $\xi$ of this recovery, which is a measure of the superconducting coherence length, is determined by fitting an exponential decay to the radial profile of the ZBTC [38],

$$
\sigma(r, 0)=\sigma_{0} e^{-r / \xi}+c,
$$

where $\sigma_{0}$ is the additional conductance in the vortex core and $c$ accounts for a constant background. We obtain $\xi=7.1 \pm$ $0.3 \mathrm{~nm}$ averaging the decay lengths obtained from the data shown in Fig. 4(a). This value of $\xi$ is close to that obtained previously for the coherence length $(10 \mathrm{~nm})$ [39].

\section{DISCUSSION}

For superconductors with an isotropic gap and which are in the clean limit, strong vortex bound states are expected near the Fermi level [40], which give rise to a strong peak in differential conductance spectra recorded in the center of a vortex core [32]. Spectra of the differential conductance acquired inside a vortex core and away from it show no evidence for a vortex bound state [see Figs. 4(b) and 4(c)]. This behavior is consistent with a not fully formed gap on some bands or a considerable amount of scattering in the superconductor (or at the surface). The mean free path in $\mathrm{CeCu}_{2} \mathrm{Si}_{2}$ is on the order of $10 \mathrm{~nm}$ [39], therefore the material is not in the clean limit.

The zero-bias conductance extracted from tunneling spectra at zero field agrees reasonably well with specific heat data of Kittaka et al. [11]: at zero field and $200 \mathrm{mK}$, our tunneling spectra yield a ZBTC of $0.74 \pm 0.05$ of the normal state conductance, whereas the normalized specific heat at $200 \mathrm{mK}$ $\left(C_{s} / C_{n}\right)$ is about 0.65 of that in the normal state. This shows that the superconducting gap we detect in tunneling spectra at the surface is consistent with what one would expect from bulk superconductivity as measured by specific heat [41].

It is interesting to compare our observations to the case of $\mathrm{CeCoIn}_{5}$, where experiments suggest a $d$-wave symmetry of the order parameter $[22,23]$. The spectra we have observed at base temperature [see Fig. 1(d)] show a striking similarity with tunneling spectra of the superconducting gap in the heavyfermion superconductor $\mathrm{CeCoIn}_{5}[22,23]$. Small angle neutron scattering of the vortex lattice in $\mathrm{CeCoIn}_{5}$ has previously shown substantial variations from triangular to square and rhombic lattice symmetries [42].

\section{CONCLUSION}

In conclusion, our tunneling spectroscopic measurements on the first discovered heavy-fermion superconductor, $\mathrm{CeCu}_{2} \mathrm{Si}_{2}$, show clear evidence for superconductivity with at least two distinct gaps, with the best fits obtained for the smaller with $d$-wave character and the larger with $s$-wave character. Although the observed gap does not open fully, the temperature and magnetic field dependent measurements establish its link to superconductivity. Characterization of the vortex lattice shows a triangular vortex lattice at low fields with significant distortion at fields around $0.7 \mathrm{H}_{c 2}$ indicating an anisotropic interaction between vortex cores. The features observed in the tunneling spectra at low energy support a multigap scenario. The shape of the gap detected in tunneling spectroscopy as well as the distortion of the vortex lattice indicate similarities with superconductivity in $\mathrm{CeCoIn}_{5}$.

Data associated with this work are available from [43].

\section{ACKNOWLEDGMENTS}

We gratefully acknowledge K. Machida and Y. Fasano for stimulating discussions. Z.S. acknowledges support from Netherlands Organisation for Scientific Research (NWO) (Grant No. 680.50.1119). A.M. and P.W. acknowledge support from the Engineering and Physical Sciences Research Council (EPSRC, EP/I031014/1).

M.E., Z.S., and A.M. contributed equally to this work. 
[1] F. Steglich, J. Aarts, C. D. Bredl, W. Lieke, D. Meschede, W. Franz, and H. Schäfer, Phys. Rev. Lett. 43, 1892 (1979).

[2] N. D. Mathur, F. M. Grosche, S. R. Julian, I. R. Walker, D. M. Freye, R. K. W. Haselwimmer, and G. G. Lonzarich, Nature (London) 394, 39 (1998).

[3] S. Doniach, Physica B 91, 231 (1977).

[4] R. Feyerherm et al., Phys. Rev. B 56, 699 (1997).

[5] S. Seiro, M. Deppe, H. Jeevan, U. Burkhardt, and C. Geibel, Phys. Status Solidi B 247, 614 (2010).

[6] O. Stockert, J. Aarndt, E. Faulhaber, C. Geibel, H. S. Jeevan, S. Kirchner, M. Loewenhaupt, K. Schmalzl, W. Schmidt, Q. Si, and F. Steglich, Nat. Phys. 7, 119 (2011).

[7] K. Ishida, Y. Kawasaki, K. Tabuchi, K. Kashima, Y. Kitaoka, K. Asayama, C. Geibel, and F. Steglich, Phys. Rev. Lett. 82, 5353 (1999).

[8] K. Fujiwara, Y. Hata, K. Kobayashi, K. Miyoshi, J. Takeuchi, Y. Shimaoka, H. Kotegawa, T. C. Kobayashi, C. Geibel, and F. Steglich, J. Phys. Soc. Jpn. 77, 123711 (2008).

[9] E. Lengyel, M. Nicklas, H. S. Jeevan, G. Sparn, C. Geibel, F. Steglich, Y. Yoshioka, and K. Miyake, Phys. Rev. B 80, 140513(R) (2009).

[10] H. A. Vieyra, N. Oeschler, S. Seiro, H. S. Jeevan, C. Geibel, D. Parker, and F. Steglich, Phys. Rev. Lett. 106, 207001 (2011).

[11] S. Kittaka, Y. Aoki, Y. Shimura, T. Sakakibara, S. Seiro, C. Geibel, F. Steglich, H. Ikeda, and K. Machida, Phys. Rev. Lett. 112, 067002 (2014).

[12] I. Eremin, G. Zwicknagl, P. Thalmeier, and P. Fulde, Phys. Rev. Lett. 101, 187001 (2008).

[13] H. Ikeda, M.-T. Suzuki, and R. Arita, Phys. Rev. Lett. 114, 147003 (2015).

[14] A. R. Schmidt, M. H. Hamidian, P. Wahl, F. Meier, A. V. Balatsky, J. D. Garrett, T. J. Williams, G. M. Luke, and J. C. Davis, Nature (London) 465, 570 (2010).

[15] P. Aynajian, E. H. da Silva Neto, C. V. Parker, Y. Huang, A. Pasupathy, J. Mydosh, and A. Yazdani, Proc. Natl. Acad. Sci. USA 107, 10383 (2010).

[16] S. Ernst, S. Kirchner, C. Krellner, C. Geibel, G. Zwicknagl, F. Steglich, and S. Wirth, Nature (London) 474, 362 (2011).

[17] P. Wahl, L. Diekhöner, M. A. Schneider, F. Treubel, C. T. Lin, and K. Kern, Phys. Rev. B 84, 245131 (2011).

[18] P. Aynajian, E. H. da Silva Neto, A. Gyenis, R. E. Baumbach, J. D. Thompson, Z. Fisk, E. D. Bauer, and A. Yazdani, Nature (London) 486, 201 (2012).

[19] A. Maldonado, I. Guillamon, J. G. Rodrigo, H. Suderow, S. Vieira, D. Aoki, and J. Flouquet, Phys. Rev. B 85, 214512 (2012).

[20] H. Sakata, N. Nishida, M. Hedo, K. Sakurai, Y. Inada, Y. Onuki, E. Yamamoto, and Y. Haga, J. Phys. Soc. Jpn. 69, 1970 (2000).
[21] H. Suderow, S. Vieira, J. D. Strand, S. Bud'ko, and P. C. Canfield, Phys. Rev. B 69, 060504 (2004).

[22] B. B. Zhou, S. Misra, E. H. da Silva Neto, P. Aynajian, R. E. Baumbach, J. D. Thompson, E. D. Bauer, and A. Yazdani, Nat. Phys. 9, 474 (2013).

[23] M. P. Allan, F. Massee, D. K. Morr, J. Van Dyke, A. W. Rost, A. P. Mackenzie, C. Petrovic, and J. C. Davis, Nat. Phys. 9, 468 (2013).

[24] C. Pfleiderer, Rev. Mod. Phys. 81, 1551 (2009).

[25] U. Singh, M. Enayat, S. White, and P. Wahl, Rev. Sci. Instrum. 84, 013708 (2013).

[26] This is likely related to the comparatively small $c / a$ ratio, which indicates a so-called collapsed structure with strong $\mathrm{Si}-\mathrm{Si}$ bonds between adjacent $\mathrm{Cu}-\mathrm{Si}$ layers.

[27] D. Ehm, S. Hüfner, F. Reinert, J. Kroha, P. Wölfle, O. Stockert, C. Geibel, and H. V. Löhneysen, Phys. Rev. B 76, 045117 (2007).

[28] See Supplemental Material at http://link.aps.org/supplemental/ 10.1103/PhysRevB.93.045123 for details on the multigap fits and a $2 \mathrm{D}$ representation of the image shown in Fig. 1(b).

[29] R. C. Dynes, V. Narayanamurti, and J. P. Garno, Phys. Rev. Lett. 41, 1509 (1978).

[30] A. E. Ruckenstein, P. J. Hirschfeld, and J. Appel, Phys. Rev. B 36, 857 (1987).

[31] H. Won and K. Maki, Phys. Rev. B 49, 1397 (1994).

[32] H. F. Hess, R. B. Robinson, R. C. Dynes, J. M. Valles, and J. V. Waszczak, Phys. Rev. Lett. 62, 214 (1989).

[33] G. Blatter, M. V. Feigelman, V. B. Geshkenbein, A. I. Larkin, and V. M. Vinokur, Rev. Mod. Phys. 66, 1125 (1994).

[34] T. A. Tokuyasu, D. W. Hess, and J. A. Sauls, Phys. Rev. B 41, 8891 (1990).

[35] Ji-Hai Xu, Yong Ren, and Chin-Sen Ting, Phys. Rev. B 53, R2991(R) (1996).

[36] M. Ichioka, N. Hayashi, N. Enomoto, and K. Machida, Phys. Rev. B 53, 15316 (1996).

[37] There is also a deviation at $0.5 \mathrm{~T}$, where, however, we observe only a few vortex cores within the field view and therefore it is difficult to extract lattice distances.

[38] S. H. Pan, E. W. Hudson, A. K. Gupta, K.-W. Ng, H. Eisaki, S. Uchida, and J. C. Davis, Phys. Rev. Lett. 85, 1536 (2000).

[39] U. Rauchschwalbe, W. Lieke, C. D. Bredl, F. Steglich, J. Aarts, K. M. Martini, and A. C. Mota, Phys. Rev. Lett. 49, 1448 (1982).

[40] C. Caroli, P. G. de Gennes, and J. Matricon, Phys. Lett. 9, 307 (1964).

[41] Y. Tsutsumi, K. Machida, and M. Ichioka, Phys. Rev. B 92, 020502 (2015).

[42] A. D. Bianchi et al., Science 319, 177 (2008).

[43] http://dx.doi.org/10.17630/ec932263-a789-435c-bbe7-8a6960 ca7358. 